\title{
Attending to two fingers: Two hands are better than one
}

\author{
JAMES C. CRAIG \\ Indiana University, Bloomington, Indiana
}

\begin{abstract}
The ability of subjects to attend to vibrotactile patterns presented to two fingers was explored in several experiments. The patterns were generated by two 6 column $\times 24$ row tactile arrays. In one set of measurements, the arrays were placed in contact with the subjects' left index and middle fingers. Both discrimination and identification tasks suggested that there was an attentional deficit in processing patterns presented simultaneously to these two fingers. A pattern presented to either the middle or index finger was identified both more accurately and more rapidly than was the same pattern divided in half and presented to two fingers. Patterns were also presented to two fingers on opposite hands, and performance measures were taken on patternidentification tasks, on discrimination tasks, and on tasks that required subjects to combine pattern information from two fingers. All three measures showed performance to improve when the patterns were presented to two fingers on opposite hands relative to when they were presented to two fingers on the same hand. The results are interpreted to suggest, first, that subjects can process patterns simultaneously presented to two fingers on the same hand, but with some deficit due to attentional mechanisms, and second, that information from patterns presented to two fingers is processed differently depending on whether the two fingers are on the same or on different hands. There is much less of an attentional deficit in processing patterns presented simultaneously to two fingers on opposite hands.
\end{abstract}

There has been relatively little work on the role of attention in the perception of tactile stimuli. In considering a task such as detection of a signal, the question of whether or not a subject can attend to more than one site has received mixed answers. Franzen, Markowitz, and Swets (1970) concluded that subjects could not attend to more than one finger at a time, whereas Craig (1968) concluded that subjects could combine weak signals from two fingers, implying that the two fingers could be attended to simultaneously. Subsequently, Shiffrin, Craig, and Cohen (1973) presented data to support an unlimited attention model for detection of simple stimuli by the skin. These studies did not, however, deal with the question of whether and how subjects could attend to patterned stimuli presented to more than one site at a time. Here the question is more than simply whether or not a stimulus was presented at a particular site; it is whether or not information from more than one site can be combined.

It has been demonstrated that subjects can use information presented to several sites on the skin at the same time, implying an ability to attend to clearly suprathreshold stimuli presented simultaneously to more than a single location. In one study, subjects were presented with pairs of patterns consisting of up to 10 vibrators, contacting 10 different sites on the body, acti-

This research was supported by a grant from the National Institutes of Health, NS-09783. The author wishes to thank Roger Rhodes for his assistance in the conduct of these experiments and Barry Green for his comments on the manuscript. The author's mailing address is: Department of Psychology, Indiana University, Bloomington, IN 47405. vated simultaneously (Geldard \& Sherrick, 1965). The fact that subjects could discriminate between pairs of the patterns demonstrated that they were able to divide their attention among widely separated sites of stimulation at the same time (or at least to switch attention rapidly from site to site). Similar results were obtained when the sites of stimulation were the fingertips (Gilson, 1968). Also, the success of Tadoma users suggests that information presented to several sites simultaneously can be combined. In Tadoma, a deaf-blind individual places his or her hand on the face of a speaker and, by means of feeling the patterns produced by the lips, jaw, and throat, is able to understand low normal rates of speech (Reed, Durlach, \& Braida, 1982). To do this, the Tadoma user is presumably sharing attention among the several sites of stimulation on his or her fingers and hand.

Some results suggesting that there may be difficulty in using information presented to two fingers were obtained by Hill (1974) in a reading task. Using the tactile displays from the Optacon (Bliss, Katcher, Rogers, \& Shepard, 1970), Hill tested a variety of display modes, including the presentation of letters to two adjacent fingers. Not finding significantly faster reading with this mode, Hill suggested that it might not be possible for information to be integrated spatially across two different fingers.

The previous work that led most directly to the present study of attention was a series of measurements of pattern discrimination. In one of these measures, pairs of patterns, of the type represented in Figure 1, were presented briefly to subjects fingerpads by means of arrays of vibrating pins. The patterns were presented to the 


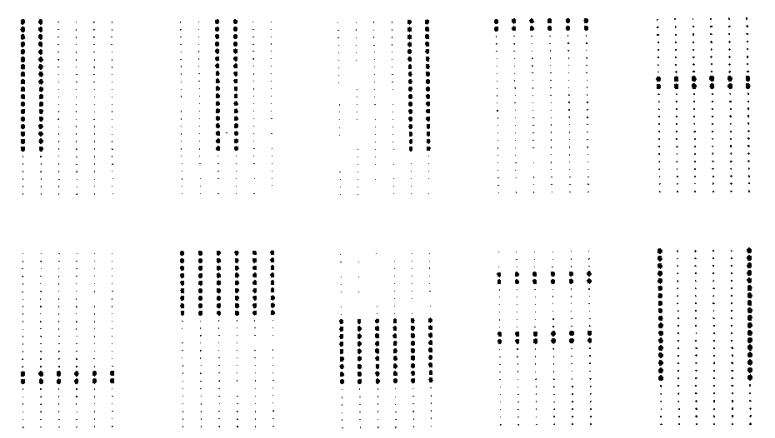

Figure 1. Representations of the 10 patterns used in the discrimination and directed-/divided-attention measurements.

index and middle fingerpads of the left hand, and the time between onsets of the two patterns, stimulus onset asynchrony (SOA), was varied.

Discrimination performance was relatively poor and remained unchanged until the SOA was greater than $100 \mathrm{msec}$, at which point it improved (Figure 15, ipsilateral function). The fact that the subjects' performances were better than $50 \%$ correct, chance performance, indicates that they could divide their attention between two fingers, although with some loss of information. The improvement in performance as the time between patterns exceeds 100 msec suggests that the subjects were able to shift their attention between the two fingers at the longer SOAs and reduce the attentional deficit.

The present study was undertaken to examine under what circumstances subjects could attend to more than a single site of stimulation, at what cost attention would be shared between two sites, how rapidly attention could be switched from one site to another, and how increasing spatial separation between two sites of stimulation would affect attention. The answers to these questions are relevant to the general issue of how the skin processes spatially and temporally extended patterns. Experiments 1, 2 , and 3 examined attention between two sites on the same hand, and Experiments 4, 5, and 6 examined attention between two sites on opposite hands.

The results of the first studies in our work on attention were discussed previously in a review article (Craig, 1985). In those studies, subjects were presented spatial patterns of the type shown in Figure 1 to both their index and middle fingerpads. On each trial, one pattern was presented to the index fingerpad and another pattern was presented to the middle fingerpad. The subject's task was to identify one of the two patterns, that is, to report on the pattern presented to one of the two fingers. On one block of trials (directed attention), the subjects were told in advance which finger to report. On another block of trials (divided attention), the subjects were not told until after both patterns had been presented which finger to report. The subjects were correct in identifying the patterns on $93 \%$ of the trials in the directed-attention condition, a percentage that fell to $77 \%$ correct in the divided- attention condition. This result indicates that subjects can divide their attention between two fingers (if they could attend to only one finger, performance would be less than $52 \%$ correct $^{1}$ ), but that they do so at some cost to the processing at each finger.

These measurements were repeated with the patterns presented successively in the divided-attention condition. The SOA was varied from 9 to $700 \mathrm{msec}$. This was done to allow subjects time to switch their attention between the middle and index fingers. The results of these measurements showed that dividing attention produced a decrement in performance and that performance changed little until the SOA exceeded $100 \mathrm{msec}$.

The fact that performance improved beyond $100 \mathrm{msec}$ indicated that it took about that long for subjects to begin to switch attention from one finger to another. The similarity between the discrimination and the directed-/dividedattention functions supports the speculation that one of the problems in discriminating between patterns presented to different fingers is that of attending to the two fingers.

\section{EXPERIMENT 1}

Experiment 1 repeated the discrimination measurements and directed-/divided-attention measurements (identification) with another, more difficult set of spatial patterns. The first reason for repeating these measurements was to see whether the discrimination and directed-/dividedattention functions would continue to parallel one another. The second reason was to examine the effect of pattern difficulty on these temporal functions. It has been suggested that attentional deficits might be more prominent with stimuli that are more difficult to discriminate (Kahneman, 1973). Would increasing the complexity of the patterns result in a change in, for example, the temporal separation at which subjects can begin to switch their attention? More difficult patterns might cause subjects to take longer than approximately $100 \mathrm{msec}$ to switch their attention from one pattern to another.

\section{Method}

Subjects. In all the experiments to be reported, the subjects were selected from a group of Indiana University students who were paid on an hourly basis for their participation. All subjects had participated in previous pattern perception tasks in the laboratory. For the discrimination measurements, 3 women and 1 man served as subjects. For the directed-/divided-attention measurements, 4 women served as subjects. One of the 4 subjects served in both sets of measurements.

Apparatus. Two tactile displays, similar to those used in the Optacon (Bliss et al., 1970), were used as tactile stimulators. Each display consists of 144 pins arranged in a rectangular array, 6 columns wide $\times 24$ rows high and measuring $11 \mathrm{~mm}$ wide $\times$ $27 \mathrm{~mm}$ high. In these studies. only the top 18 rows, or $20 \mathrm{~mm}$ were used. The arrays are designed to fit against the distal portion of a subject's fingerpad, and each pin can be made to vibrate at $230 \mathrm{~Hz}$. The arrays were interfaced with a PDP-11/34 computer, which controlled the presentation of stimuli and recorded subjects' responses. The same apparatus was used for all experiments reported here. Additional information about the apparatus may be found in Craig (1980). 
Procedure. Subjects were seated with their left hands extended. Their left index fingerpads rested on one of the two tactile displays, and their left middle fingerpads rested on the other tactile display. The subjects responded with their right hands, using a keyboard.

One group of subjects performed a discrimination task; the other performed an identification task. For both tasks, the stimuli were the 26 uppercase letters of the alphabet displayed on the top $20 \mathrm{~mm}$ of the tactile arrays. Examples of these stimuli are shown in Figure 2. In the discrimination task, the pairs of letters were selected randomly such that on half the trials the two patterns making up the pair were the same and on half the trials they were different. Trial-by-trial feedback was provided. The SOA was varied from 0 to 400 msec. Specifically, seven blocks of trials, 42 trials per block, were run. Randomly presented within a block of trials were seven SOAs $-0,9,26,52,100,200$, and $400 \mathrm{msec}$. The pattern presented to the middle finger always preceded the pattern presented to the index finger.

For the directed-/divided-attention task, subjects were presented pairs of letters and required to identify one member of the pair. On half the trials, the subjects attempted to identify the pattern presented to the middle fingerpad, and on the other half of the trials, the subjects attempted to identify the pattern presented to the index fingerpad. The two conditions of the experiment, directed- and divided-attention conditions, were similar to those described before (Craig, 1985). In the directed-attention condition, the subject was informed, by means of a CRT, which finger to attend to. In the divided-attention condition, the subject was not informed until $500 \mathrm{msec}$ after the second pattern had been presented which finger to attend to. In the divided-attention conditions, the patterns were presented sequentially, with the pattern presented to the middle fingerpad preceding the pattern presented to the index fingerpad. SOAs of $0,9,26,52,100,200$, and $400 \mathrm{msec}$ were tested. In the directedattention condition, the two patterns were presented simultaneously, that is, at 0 -msec SOA. Trials were presented in 30-trial blocks and, in the divided-attention condition, blocked by SOA. In the directed-attention conditions, one block of trials was devoted to the middle finger and another block to patterns presented to the index finger. The blocks were presented in random order.

For both the discrimination and directed-/divided-attention tasks, the patterns were presented for $52 \mathrm{msec}$, with the intensity of the vibration on the tactile array set at a comfortable level. This level remained unchanged throughout the course of the experiment. The subjects wore earphones through which white noise was fed to eliminate any auditory cues produced by the tactile array.

\section{Results and Discussion}

The results of the discrimination measurements are based on 672 trials per point, 168 from each of 4 subjects (Figure 3 ). The results of the identification measurements are based on 720 trials, 180 from each of 4 subjects (Figure 4).

As in our previous measures of discrimination performance, a signal detection theory measure of performance
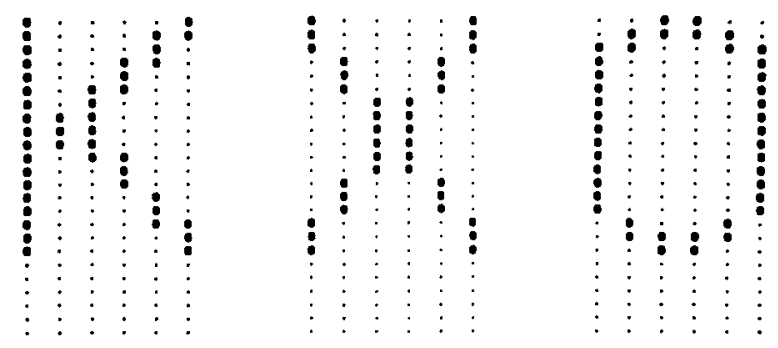

Figure 2. Representations of the types of patterns used in Experiment 1.

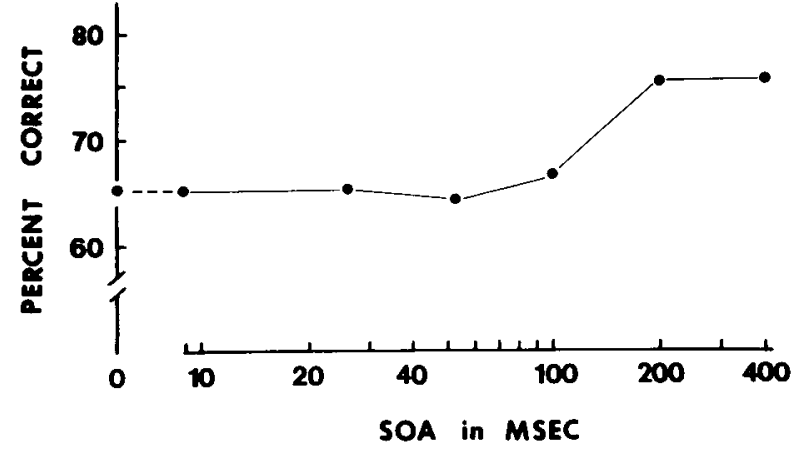

Figure 3. Percent correct discrimination plotted as a function of stimulus onset asynchrony (SOA).

was used. Trials on which subjects responded "different" when the members of the pair were different were considered "hits." Responding "different" when the members of the pair were the same was considered a "false alarm." With the hit and false-alarm rates thus obtained, d' was computed for each SOA from a computer program (Cholewiak \& Craig, 1984), and from each d' a P(C) max was computed, the proportion correct the subject would have achieved had the subject shown no response bias (McFadden, 1970). $P(C)$ max is plotted as a function of SOA in Figure 3. As was true of our previous discrimination measurements, subjects did show a decided bias toward responding "same" at the briefer SOAs. Some possible reasons for this will be considered later in this paper.

The standard errors of the means were computed on the "same" responses and "different" responses by summing across sessions and subjects for each SOA. These values ranged from $2 \%$ to $5 \%$.

Comparing Figures 3 and 4 with Figure 10 in Craig (1983) and with Figure 16 in Craig (1985) shows that, as expected, the letter patterns were more difficult both to discriminate and identify than were the previous patterns. However, even with a different and more difficult set of patterns, the function relating performance to SOA in a discrimination task is similar to the divided-attention function. The results suggest that the original observation of the similarity between the discrimination and directed-/divided-attention functions was not due to the patterns selected. In addition, a temporal separation greater than approximately 50 to $100 \mathrm{msec}$ appears to be necessary for performance to improve, the same separation seen with the less difficult patterns used before. If these results do reflect the time required to begin switching attention from one finger to another, then such switching appears to be relatively independent of pattern difficulty. It does not take longer to switch attention between patterns simply because the patterns are more difficult to perceive.

Performance levels in the divided condition, even at an SOA of $400 \mathrm{msec}$, have still not risen to the level of directed performance. Additional temporal separation would likely be required before performance in the 


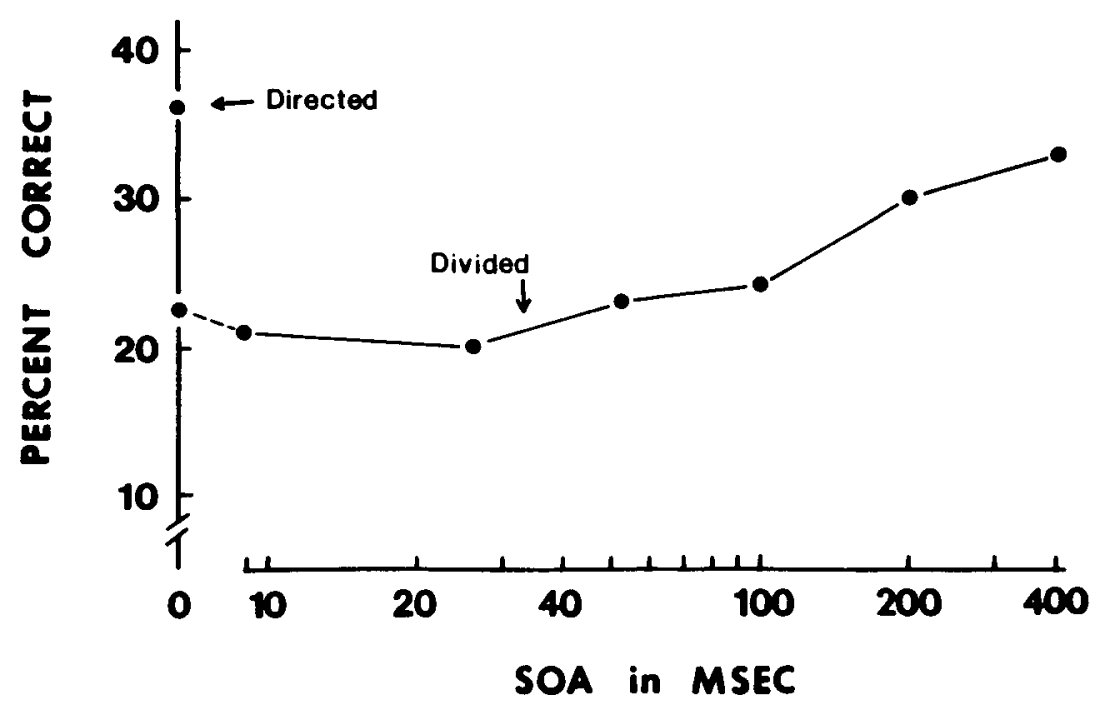

Figure 4. Percent correct identification in the directed-/divided-attention task as a function of SOA. The point marked "directed" refers to the condition in which subjects were informed in advance which finger to attend to; "divided" refers to the condition in which subjects were not told until after the patterns had been presented which finger to report on.

divided-attention condition would equal that achieved with directed attention. These results indicate that a considerable temporal separation is required before two tactile patterns can be processed independently, even when the two patterns are presented on separate fingers.

\section{EXPERIMENT 2}

Although they make only one response, subjects in the divided-attention conditions are, in fact, required to identify two patterns. In understanding speech by means of Tadoma or in recognizing an object by touch, a person must be aware of different patterns in contact with the fingers; the index finger might be in contact with a sharp edge while the middle finger is in contact with a shallow groove. Often, however, a person is trying to integrate or combine the patterns coming from the separate fingers in order to make a single response, without trying to identify the separate patterns. Experiment 2 compared performance of subjects in identifying a single spatial pattern when that pattern was presented to one finger with performance in the condition in which the pattern extended over two fingers.

In earlier work, it had been speculated that patterns presented to a single fingerpad were processed in parallel whereas patterns presented to larger body surfaces were processed serially (Craig, 1981, 1983). Serial processing presumably requires the subject to shift attention from one part of a pattern to another. In the present experiment, a decline in performance when patterns were extended over two fingers would support the hypothesis that subjects were processing the patterns serially.

\footnotetext{
Method

Subjects. Two women and 2 men served as subjects.
}

Procedure. The subjects' task in Experiment 2 was to identify which one of nine patterns had been presented. Representations of the patterns are shown in Figure 5. After each presentation of a pattern, the subjects responded by pressing one of nine keys marked with visual representations of the patterns. The patterns were divided along the vertical axis such that both halves of the patterns needed to be perceived for identification, and the pattern could be shifted such that half of it was presented on one display and half on the other display (Figure 6).

On one block of trials, the patterns were presented on the left index fingerpad; on another block of trials, the patterns were presented on the left middle fingerpad; and on a third block of trials, the patterns were split and presented to both fingers, as shown in Figure 6 . The blocks of trials, 50 trials per block, were run in random order. A testing session consisted of six blocks, two repetitions of the three experimental conditions.

\section{Results and Discussion}

The results, based on a total of 4,000 trials, 1,000 from each of 4 subjects, are shown in Figure 7: When the patterns were presented to the index fingerpad, the percent correct was $83 \%$; with the middle fingerpad, it was $77 \%$. When the patterns were split between the two fingers, performance dropped to $58 \%$. Standard errors of the means ranged from $1 \%$ to $2 \%$. A Wilcoxon signed-ranks test showed that when patterns were presented to both fingers, performance was significantly below that achieved on the poorer of the two fingers, that is, the middle finger $(p<.001)$.

If subjects could attend to only a single finger, then performance in the two-finger condition would be no higher than $33 \%$ correct. This assumes that the subject was able to perceive with $100 \%$ accuracy the half of the pattern presented to the finger to which they were attending and then randomly selected among the three patterns that contained the half they perceived. The conclusion to be drawn from this task, in which subjects make a single response 

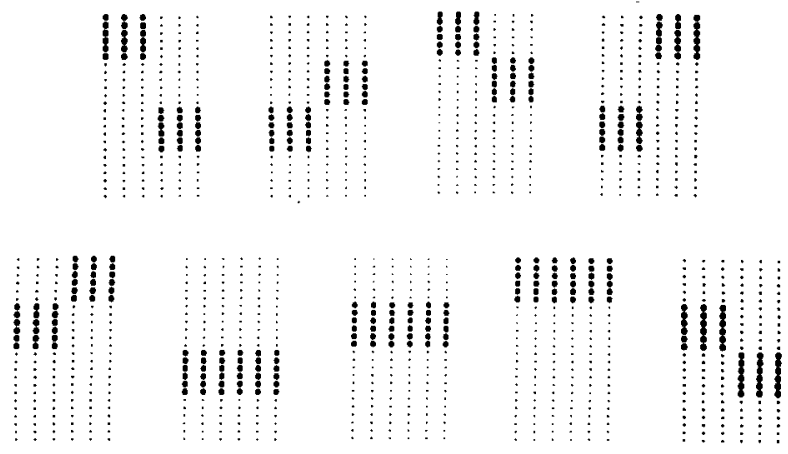

Figure 5. Representations of nine patterns used in Experiment 2.

to a divided pattern, appears to be the same as that drawn from the discrimination and directed-/divided-attention measures. Subjects can attend to more than one finger; however, performance is better if subjects do not have to share attention between two fingers. Put another way, the results suggest that subjects are processing patterns extending across two fingers serially, whereas confining the pattern to a single finger permits the pattern to be processed in a parallel fashion. Experiment 3 followed up on this suggestion by seeing if it would take measurably longer to process a pattern extending across two fingers than to do so if the pattern was presented to a single finger.

\section{EXPERIMENT 3}

Experiment 2 showed that recognition performance declined when patterns extended across two fingers, a result that was interpreted to reflect the subjects' difficulty in attending to two fingers. The results could also be interpreted to suggest that subjects were processing the two halves of a split pattern successively by attending to one finger and then the other and finally combining the information from the two fingers. This successive processing of the patterns presented to the two fingers would require more time than that required by the same pattern presented to a single finger. It is true, however, that when the two halves of the patterns are presented simultaneously, performance is well above chance on the split patterns. The latter observation suggests that subjects may be able to process the two halves of a split pattern simultaneously.

Similarly, the results from the directed-/dividedattention measurements could be interpreted to support either simultaneous or successive processing of patterns presented to two fingers. Performance at the briefest SOAs is well above chance, indicating some simultaneous processing. However, performance improves as the time between pattern onsets increases beyond $100 \mathrm{msec}$, suggesting that successive processing of the two patterns may be the preferred mode. To examine the question of processing time, Experiment 3 repeated the measurements made in Experiment 2 and measured the reaction time for correct identification of the patterns. The pattern set was changed to vary the level of difficulty of the task.

\section{Method}

Subjects. Four women were tested.

Procedure. The procedure was similar to that followed in Experiment 2. As in Experiment 2, the subjects' task was to identify patterns presented to the left middle finger, the left index finger, or split between the two fingers. The subjects were told that their reaction times to these patterns would be measured and were instructed to respond as rapidly and accurately as possible.

Two sets of patterns were tested. In one set were the same nine patterns used in Experiment 2. The other set, and the one tested first in this experiment, consisted of four patterns divisible along the vertical axis. These patterns are shown in Figure 8.

As in Experiment 2, on one block of trials, the patterns were presented to the index fingerpad, on another block, they were presented to the middle fingerpad, and on a third block, the patterns were split between the two fingers. The patterns were presented for 26 msec. Each block consisted of 50 trials. Six blocks of trials, with the three conditions presented twice in random order, comprised a testing session. Testing with the set of four patterns was completed before testing with the nine patterns was begun.

\section{Results and Discussion}

The percent correct recognition results with both the four- and nine-pattern sets are shown in Figure 9. Each point represents the results of 2,400 trials, 600 from each of 4 subjects. The nine-pattern measurements, which constitute a replication of Experiment 2, produced results very similar to those seen in Experiment 2 . The ninepattern results are based on 2,800 trials, 700 from each of 4 subjects. The standard errors of the means for both sets of patterns ranged frcm $1 \%$ to $2 \%$.

For both sets of patterns, the condition in which the pattern was split between the two fingers leads to levels of performance significantly below that achieved by the poorer of the two fingers $(p<.01$ for both sets of patterns, Wilcoxon signed-ranks test). With the nine-pattern set, performance on the middle finger and on the index finger averaged $84 \%$ correct; performance declined by $30 \%$ to $54 \%$ correct in the split condition. For the fourpattern set, performance on the middle finger and index finger averaged $97 \%$; this declined by $13 \%$ to $84 \%$ correct in the split condition. Because of the potential problem of a ceiling effect with the four-pattern set and higher rates of chance performance, not too much can be made of what
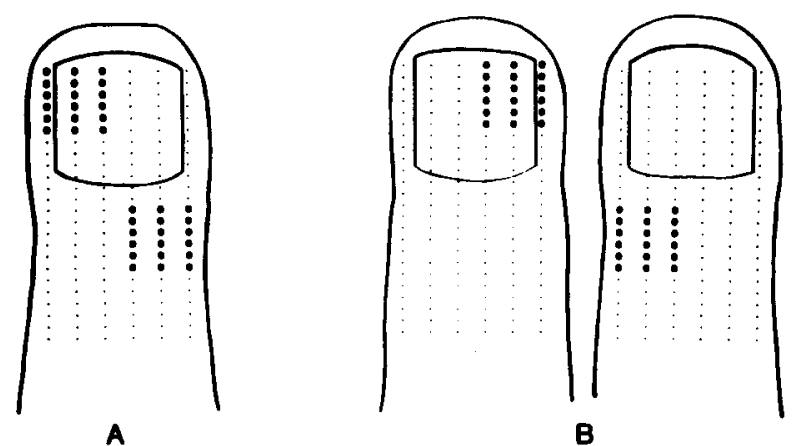

Figure 6. Representation of the manner in which a pattern was presented to a single finger (Panel $A$ ) or split between two fingers (Panel B). 


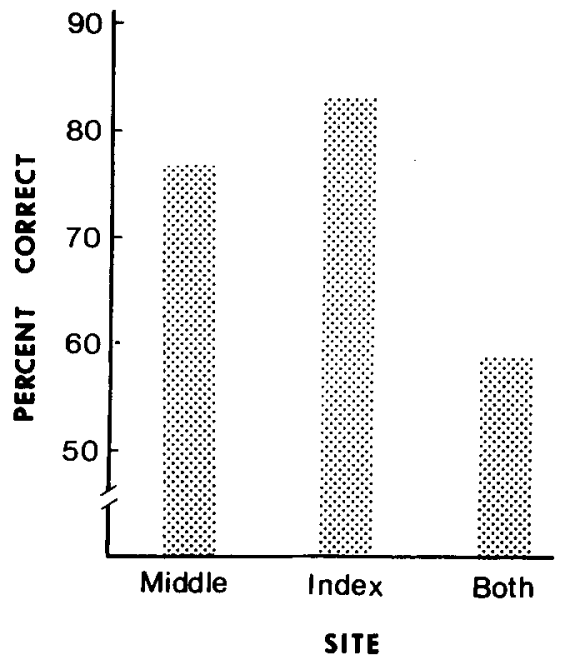

Figure 7. Identification performance for patterns presented to either the middle or index finger or split between both fingers.

might appear to be a greater attentional deficit with more difficult patterns.

The reaction time results shown in Figure 10 are based on only those trials in which the subject correctly identified the pattern. The number of trials represented in Figure 10 ranges from a low of 1,519 to a high of 2,444. The standard errors of the means ranged from 64 to 109 msec.

The results show that it takes significantly longer to respond correctly to a pattern split between two fingers than it does to respond correctly to a pattern presented to a single finger $(p<.01$, Wilcoxon signed-ranks test, for both the nine- and four-pattern sets). The average increase in reaction time in the split condition for the fourpattern set is $166 \mathrm{msec}$; the corresponding figure for the nine-pattern set is $160 \mathrm{msec}$.

Should the increase in reaction time in the split conditions, approximately $160 \mathrm{msec}$, be taken as the amount of time required to combine the information from the two fingers, or do these measurements simply reflect the fact that reaction times are longer with more difficult patterns, that is, those in the split condition? Comparing the reaction times from the nine-pattern set with those from the four-pattern set shows that reaction times to more difficult patterns are longer; it takes an average of $355 \mathrm{msec}$ longer for subjects to respond to the nine-pattern set. On the other hand, the fact that the absolute increase in reaction times in the split conditions is about the same for both sets of patterns suggests that this increase, $160 \mathrm{msec}$, is the amount of time required to combine patterns from the two fingers. Although the reaction time results were based on trials in which the subject correctly identified the pattern, the main effect, an increase in reaction time in the split condition, holds for results on the incorrect trials as well. Also, incorrect reaction times are longer than their comparable correct reaction times for all six conditions.

\section{EXPERIMENT 4}

Experiment 3 showed that increasing the spatial separation between pattern elements, that is, splitting a pattern and extending it across two fingers, increased both the difficulty in identifying the pattern and the time required to process the pattern. These results suggest that one might undertake a systematic manipulation of distance between two spatial patterns on the skin and observe any change in the ability of subjects to attend to these patterns. Unfortunately, our current instrumentation does not permit such an exploration; however, we can extend the measures of attention by separating by a considerable distance the two patterns to be presented. This can be achieved by presenting the patterns to the fingerpads on two hands, that is, bilaterally.

As the two hands are ordinarily used to explore objects, two fingers on the same hand, particularly if they are adjacent fingers, tend to receive more similar patterns than the fingers on opposite hands. Also, the neural pathways originating from the same hand are much more closely related spatially to one another than are connections

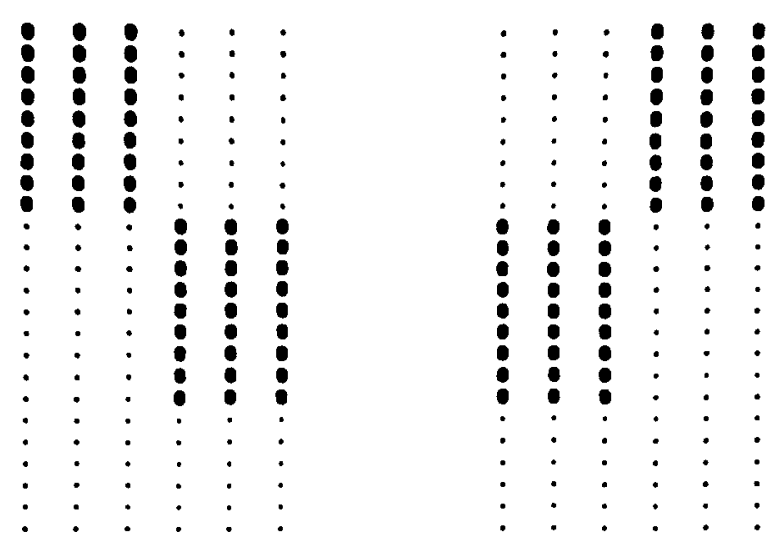

Figure 8. Representations of one set of patterns used in Experiment 3 . 


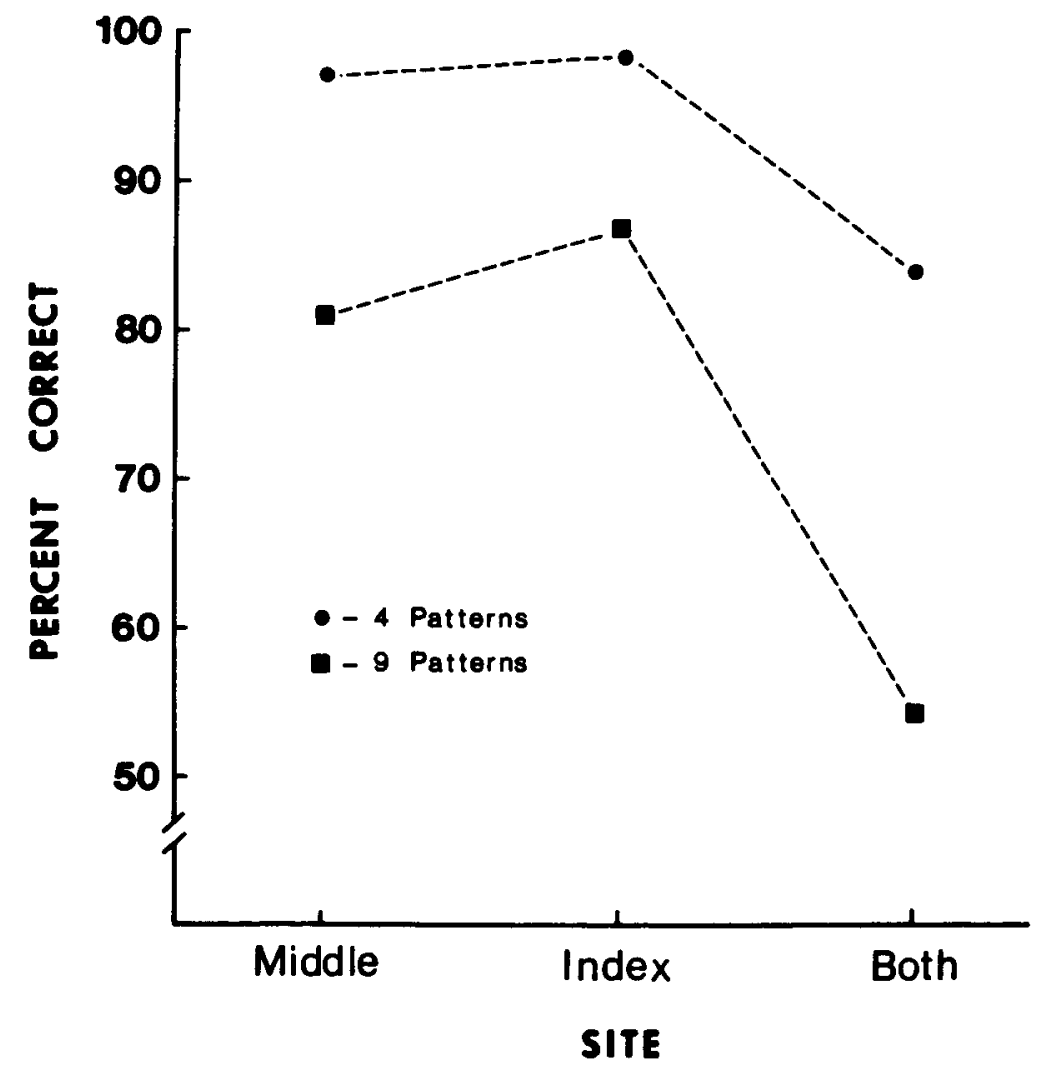

Figure 9. Identification performance for patterns that were presented to either the middle or index finger or were split between both fingers. Pattern sets consisting of four and nine patterns were tested.

originating from the other hand. These considerations would lead one to predict that it would be easier to attend to two fingers on the same hand than to two fingers on different hands.

There are few studies that bear directly on the issue of bilateral attention. Hill (1974) examined the ability of subjects to read using Optacon displays when these displays were in contact with the two index fingers. Performance in this condition was no better and no worse than using just the left index fingerpad. Hill concluded that, for practiced subjects who have been trained to read with the left index fingerpad, adding the right index fingerpad has no effect on performance. Hill's results do not make it clear whether or not subjects might be able to use information from the two index fingers if performance levels at the two fingers were more similar.

One behavioral measure that suggests that improvement might be seen using fingers from two hands comes from a study of braille reading by Lappin and Foulke (1973). In their study, subjects identified braille cells with one finger, two fingers, or four fingers. Performance did not improve as the number of fingers increased, except when the two index fingers on opposite hands were used. A second behavioral measure consistent with the Lappin and Foulke results comes from our laboratory (Craig, Green,
\& Rhodes, 1985). Vibrotactile patterns derived from speech signals (Green, Craig, \& Pisoni, 1983; Green, Craig, Wilson, Pisoni, \& Rhodes, 1983) were divided in half and presented to two fingerpads. For one group of subjects, the patterns were presented to fingers on the same hand; for a second group, the patterns were presented to fingers on opposite hands. It was the latter condition, using two fingers on opposite hands, that led to better identification of the patterns (Craig et al., 1985).

In Experiment 4, we used two measures, directed/ divided attention (Experiment 1) and combining patterns (Experiment 2), to examine attention to two fingers on opposite hands.

\section{Method}

Subjects. Three women and 1 man served as subjects.

Procedure. The procedure for the directed-/divided-attention measurements was similar to that used in Experiment 1. On each trial, subjects were presented with a pair of patterns selected from among 10 easily identified patterns (Figure 1), one member of the pair to one fingerpad and the second member to another fingerpad. In one condition of the directed-/divided-attention measurements. the patterns were presented to the subject's left middle fingerpad and left index fingerpad (ipsilateral condition). In the second condition, the patterns were presented to the left middle fingerpad and the right index fingerpad (bilateral condition). For both conditions, the subject responded verbally and the response was recorded by 


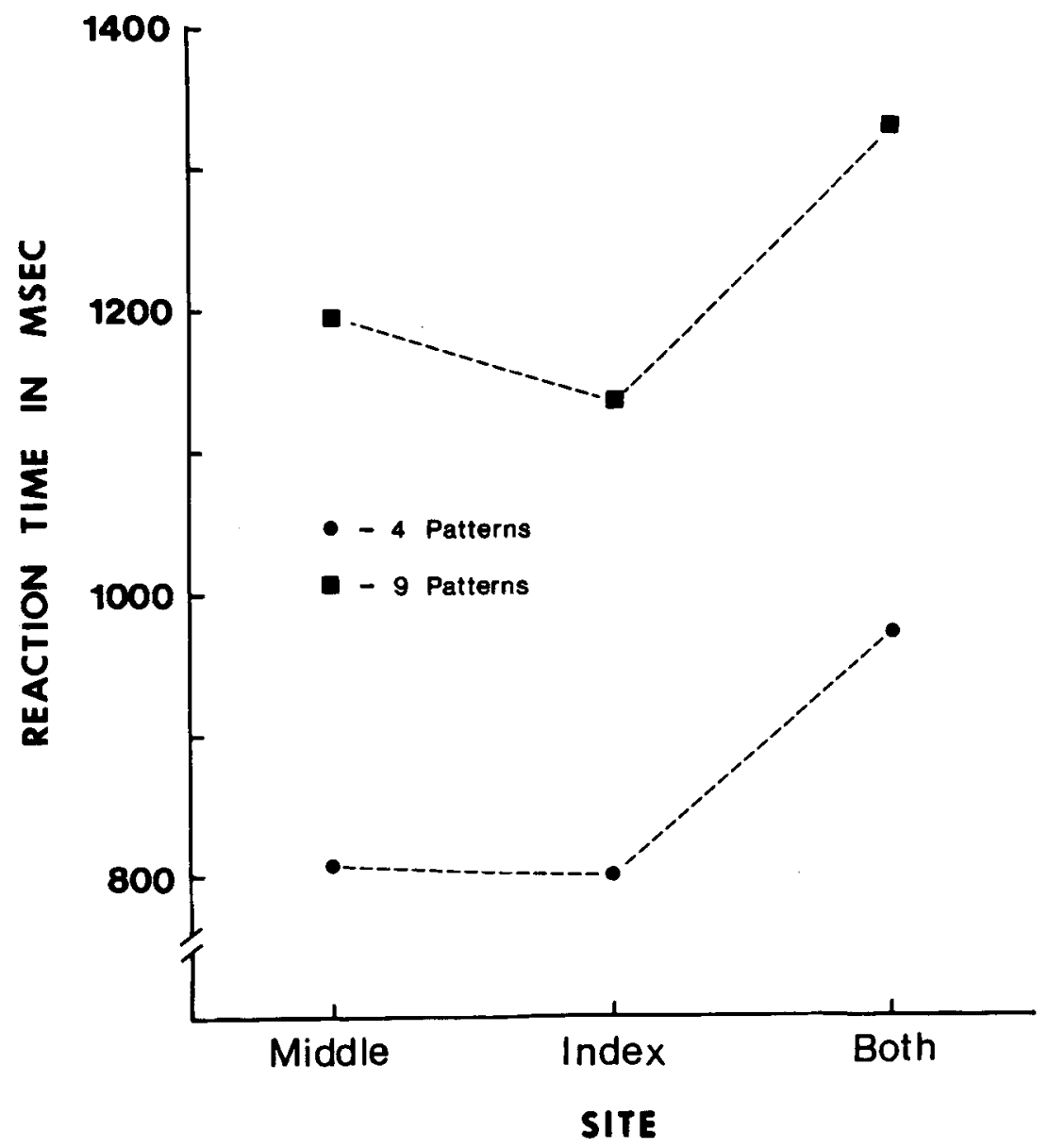

Figure 10. Reaction time for correct identification of patterns that were presented to either
the middle or index finger or were split between both fingers. Pattern sets consisting of four
and nine patterns were tested.

an experimenter. The onset of the middle-finger patterns preceded the index-finger patterns by $9,52,100,200,400$, and $800 \mathrm{msec}$. The patterns were presented for $52 \mathrm{msec}$.

Testing sessions alternated between ipsilateral and bilateral conditions and consisted of eight blocks of 40 trials each. Six of the blocks were devoted to divided attention, one block at each of the six SOAs; two blocks were devoted to directed attention, one block for the middle and one for the index fingerpads. The blocks were run in random order.

The procedure for the combining task was similar to that described in Experiment 2. The subject's task was to identify which one of nine patterns (Figure 5) had been presented. The patterns were presented one at a time for $26 \mathrm{msec}$, a duration selected to be comparable to the previous combining measurements. Patterns were presented to either the left index fingerpad or the right index fingerpad, or were split such that half of each pattern was presented to the two index fingerpads. The subjects responded verbally and received trial-by-trial feedback. A testing session consisted of six blocks of 50 trials each.

\section{Results and Discussion}

The results from the directed-/divided-attention task are presented in Figure 11. The points marked "directed" represent the mean performance on the middle and index fingers in the directed-attention condition and are the result of 1,280 trials, 320 trials from each of 4 subjects. All the remaining data points represent the divided-attention condition, and each point represents the results of 640 trials, 160 trials from each of 4 subjects. The standard errors of the means in the bilateral condition ranged from $.5 \%$ to $1.5 \%$, whereas in the ipsilateral condition they ranged from $.8 \%$ to $4.1 \%$. The smaller variability in the bilateral condition may be the result of a ceiling effect.

The ipsilateral results replicate the results of Experiment 1 in that, first, there is a decline in performance when subjects attempt to attend to patterns presented to two fingers and, second, subjects can begin to switch attention from one finger to another at SOAs greater than about 50 to $100 \mathrm{msec}$ SOA. The bilateral results provide quite a different picture. First, there is only a small decline in performance when subjects are required to divide attention between two fingers; and, second, performance is unaffected by increasing the time between the patterns.

The results from the combining pattern measurements are shown in Figure 12. These bilateral results represent 


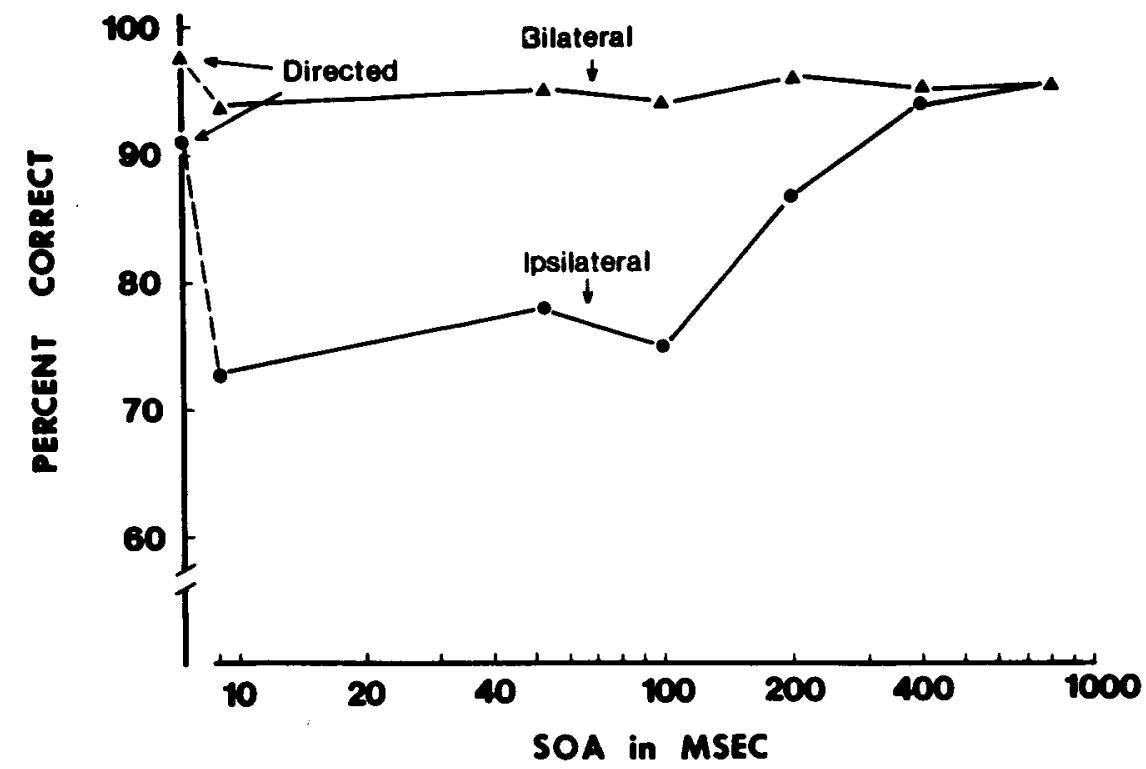

Figure 11. Percent correct identification in a directed-/divided-attention task as a function of SOA. Similar to Figure 4. In the bilateral condition, the patterns were presented to the left middle and right index fingerpads. In the ipsilateral condition, the patterns were presented to the left middle and left index fingerpads.

2,000 trials, 500 from each of 4 subjects. The standard errors of the means were approximately $2 \%$. As in the ipsilateral condition in Experiment 2, presenting the pattern to two fingers bilaterally results in a significant reduction in performance (Mann-Whitney $U$ test, $p<.02$ ); but, contrary to our expectations, it appears to be less difficult to combine patterns bilaterally than to do so ipsilaterally. The performance levels were significantly better when patterns extended across both fingers bilaterally than when they did so ipsilaterally (Figure 7) under similar conditions (Wilcoxon signed-ranks test, $\mathrm{p}<.01$ ).

The main conclusion to be drawn from both sets of measurements is that when spatial patterns are presented to two fingers they are more easily processed when the two fingers are on opposite hands than when they are on the same hand. This difference in perceptibility we would like to attribute to subjects' being able to attend more easily to bilateral than to ipsilateral patterns. In the directed- $/$ divided-attention measurements, for example, subjects do about as well in identifying patterns bilaterally whether they are told in advance which finger to attend to or are not told until $500 \mathrm{msec}$ after the patterns have been presented. Such was not the case for ipsilateral patterns.

Figure 11 also shows that performance in the ipsilateral directed condition is lower than the comparable performance in the bilateral condition. This lower performance is due to the fact that performance was poorer on the left middle finger than on the right index finger and is most likely due to masking of the patterns presented to the middle finger by the patterns presented to the left index finger. The fact that performance at the longer SOAs, 400 and $800 \mathrm{msec}$-temporal separations at which masking is diminished (Craig, 1983) - rises above directed performance suggests that masking is playing a role, a factor that will be considered in the General Discussion.

\section{EXPERIMENT 5}

Experiment 5 examined the role of limb placement in attending to spatial patterns. Following a line of reasoning stated earlier-the closer together two fingers are, the more likely they are to encounter the same pattern-we repeated the bilateral measures of attention with the two tactile arrays adjacent to one another (the subject's index fingers placed close together) and with the two arrays placed about $1.5 \mathrm{~m}$ apart (the subject seated with arms extended).

\section{Method}

Subjects. Four subjects were tested. Three of the subjects were women, and 1 was a man.

Procedure. The two measures used in Experiment 4, combining patterns and directed/divided attention. were repeated under two conditions of limb placement. In one condition, the two tactile arrays were placed adjacent to one another so that the subjects' arms were parallel and directly in front of them. In the second condition, the arrays were placed approximately $1.5 \mathrm{~m}$ apart so that the subjects had to extend their arms to the side and forward slightly to contact the arrays. The arrays were turned (the angle formed by two arrays was slightly less than $180^{\circ}$ ) to permit comfortable placement of the subjects' hands and fingers. In the combining pattern tasks, two placements of the arrays, together or apart, were tested within a single session, with half of the sessions beginning with the arrays placed together and the remaining sessions with the arrays apart. Each session consisted of 300 trials, six blocks of 50 trials each. In the directed-/divided-attention task, the placements, 


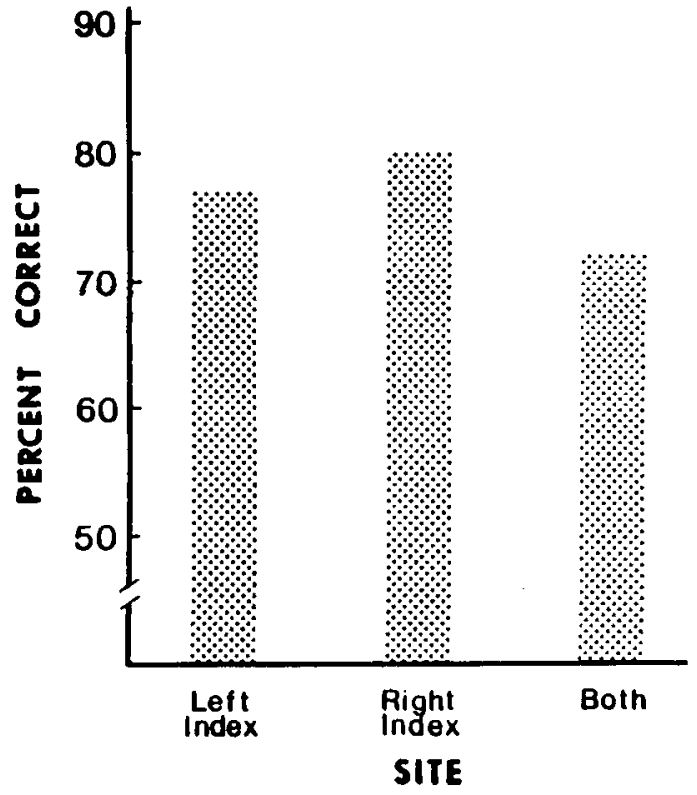

Figure 12. Percent correct indentification for patterns that were presented to either a single finger or were split between two fingers on opposite hands.

together or apart, were alternated between sessions. Each session consisted of 320 trials, eight blocks of 40 trials each. The SOAs in the divided-attention condition were 9, 52, 100,200,400, and $800 \mathrm{msec}$. In both sets of measurements, the left and right index fingerpads were the sites of stimulation.

\section{Results and Discussion}

The results from the combining pattern task are shown in Figure 13. Each entry represents 1,200 trials, 300 from each of 4 subjects. The standard errors of the means ranged from $1 \%$ to $3 \%$. There is a significant decline in performance resulting from splitting the patterns between two fingers when the hands are together $(p<.001$, Wilcoxon signed-ranks test), a result that replicates the findings in Experiment 4. When the two hands are apart, the decline in performance fails to reach significance at the .01 level; the decline is, however, significant at the .05 level (Wilcoxon signed-ranks test). The difference beween the two performance levels when the patterns extend across both fingers fails to reach significance $(p>.05$, Wilcoxon signed-ranks test). The conclusion to be drawn from these measurements is that it is no more difficult to combine information from two hands when the hands are extended than when the hands are together, and, in fact, it may even be somewhat easier to combine patterns.

The results from the directed-/divided-attention measurements are shown in Figure 14. The points marked "directed" represent 960 trials, 240 from each of 4 subjects, and are mean responses from the left and right index fingers in the directed-attention condition. The remaining data points, those in the divided-attention condition, are the result of 480 trials, 120 from each of the 4 subjects. The standard errors of the means ranged from $1 \%$ to $2 \%$.
In the directed-attention condition, there is virtually no decline with the arms apart and only a slight decline with the arms together. In fact, there is a significant decline in performance in this latter condition $(p<.01$, Wilcoxon signed-ranks test). However, calculating the mean performance across SOAs in the divided-attention condition shows less than a $1 \%$ advantage in having the arms apart over having them together. The combined pattern measurements and directed-/divided-attention measurements suggest that the processing of tactile patterns is affected only slightly, if at all, by limb placement. And, to the extent that there is a difference due to limb placement, it is in the direction of there being less of an attentional deficit with the arms extended.

\section{EXPERIMENT 6}

If attention is playing a major role in discriminating between patterns presented to two fingers on the same hand, and if presenting patterns bilaterally reduces the attentional deficit, then bilateral pattern discrimination ought to be considerably better than ipsilateral pattern discrimination. A fairly specific prediction can be made: Bilateral presentation of the patterns should produce an overall improvement in the level of performance, as compared with ipsilateral presentation, and the performance should remain unchanged as SOA is increased.

\section{Method}

Subjects. Four subjects, all women, were tested on the 10-pattern discrimination. On the 26 -letter discrimination, 5 subjects, all women, were tested.

Procedure. Two sets of bilateral discrimination measurements were made, one with the 10 easy patterns (used in Experiment 4) and the other with 26 letters. The patterns were presented to the left index finger and to the right index finger.

On each trial using the 10 patterns, the subjects received a pair of patterns drawn from the set of 10 patterns. On half the trials, the patterns were the same; on the other half, the two patterns were different. At the end of the trial, the subjects responded either "same" or "different" and were told whether or not they were correct. The SOAs were set at $0,9,26,52,100,200$, or $400 \mathrm{msec}$. The trials were not blocked by SOA. Each member of the pair of patterns was presented for $52 \mathrm{msec}$. The pattern presented on the left display preceded the pattern presented on the right display.

The major difference between the 10-pattern measurements and the 26-letter measurements was simply the number and difficulty of the patterns from which the pairs were drawn. The letters are more difficult to recognize than the 10 patterns, and the number of possible pairs is considerably larger. The same SOAs used with the 10 patterns were used with the letter patterns.

\section{Results and Discussion}

The 10-pattern discrimination data were analyzed as in Experiment 1 , and $\mathrm{P}(\mathrm{C})$ max was computed. It is this corrected percent correct that is plotted in Figure 15.

Each point on the curve depicting the bilateral results in Figure 15 represents 672 trials, 168 from each of 4 subjects. For comparison purposes, ipsilateral discrimination results using two fingers on the same hand (Craig, 1983) are plotted as well. As predicted, bilateral discrimination is considerably better than ipsilateral discrimina- 


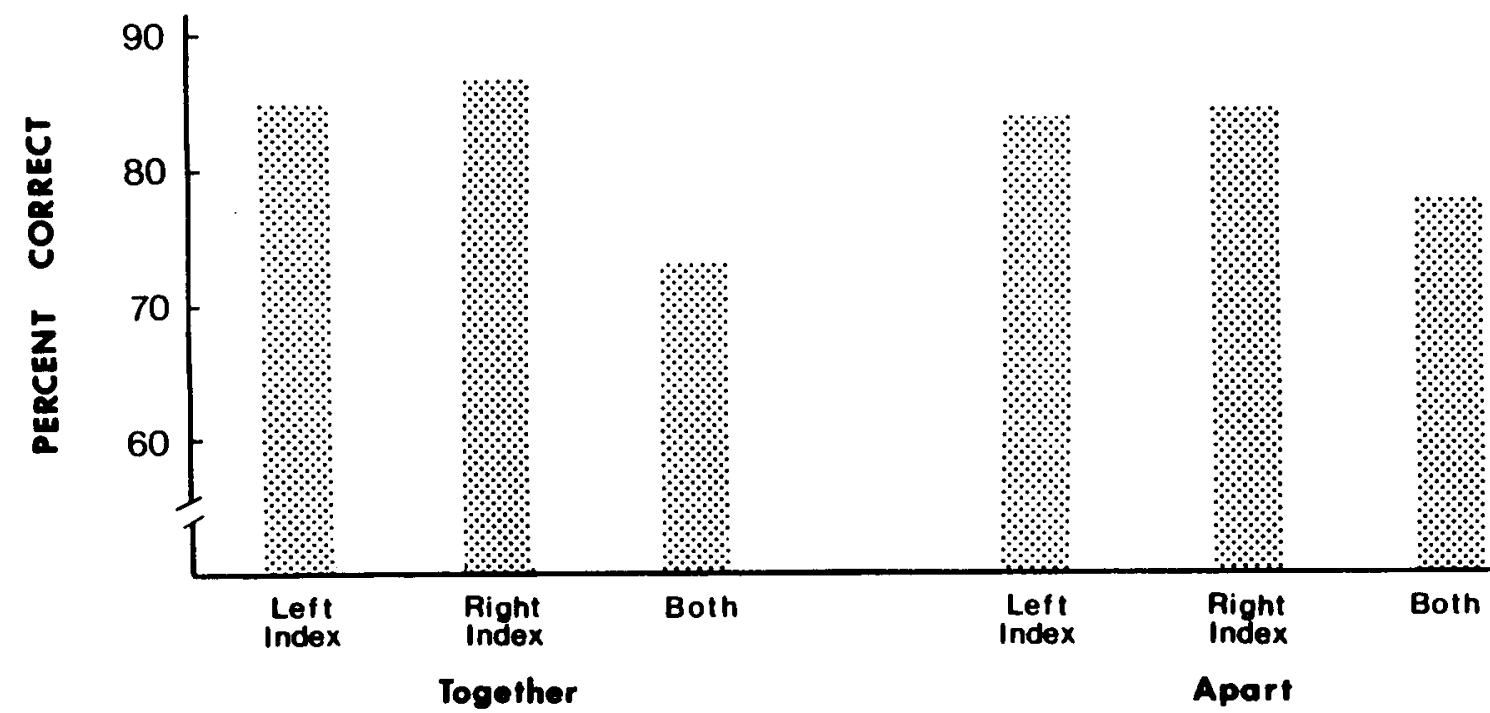

\section{ARM PLACEMENT}

Figure 13. Similar to Figure 12. "Together" refers to positioning arms close together and contacting the two tactile arrays placed next to one another. "Apart" refers to positioning the arms to the side and contacting the two tactile arrays placed about $1.5 \mathrm{~m}$ apart.

tion and appears to be unaffected by changes in SOA. Also shown in Figure 15 are results from discrimination measurements made when both patterns were presented to the same finger (Craig, 1983).

For patterns presented to a single finger, the function relating discrimination performance to SOA has been attributed to masking. Performance gradually improves because the amount of temporal masking is reduced (Craig, $1978,1980,1983$ ). Separating the pair of patterns spatially by moving one of the patterns to a second finger reduces masking considerably (Craig, 1983), yet performance at the briefer SOAs for the two-finger ipsilateral condition does not improve and is, in fact, lower than that in the single-finger condition. It was for this reason, in part, that we attributed the poor ipsilateral performance to problems in attending to two locations simultaneously. As Figure 15 also shows, the bilateral condition when both masking and, presumably, the attentional deficit are reduced leads to better discrimination than either the single-finger condition or the two-finger ipsilateral condition.

A similar picture emerges with the more difficult patterns. The 26-letter discrimination results are shown in Figure 16. For the bilateral data, each point represents 840 trials, 168 from each of 5 subjects. The ipsilateral data from Figure 3 are shown for purposes of comparison. Discrimination performance is better in the bilateral condition, and SOA does not appear to have a consistent effect on discrimination. However, examination of just the portion of the function from an SOA of $52 \mathrm{msec}$ out

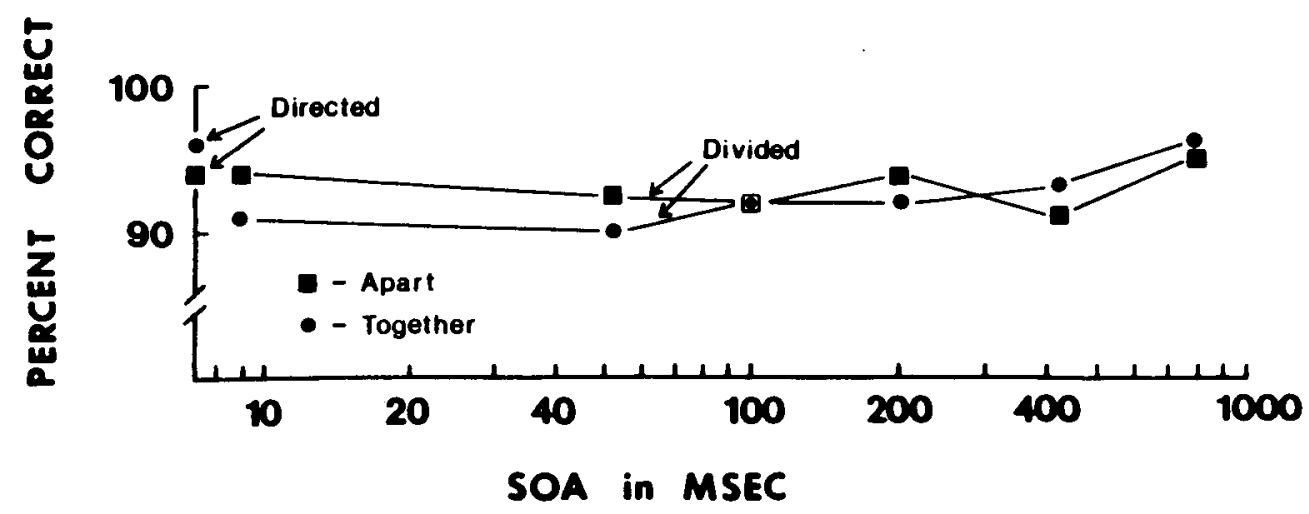

Figure 14. Similar to Figure 4. "Apart" and "together" refer to the positioning of the arms and tactile arrays, as in Figure 13. 


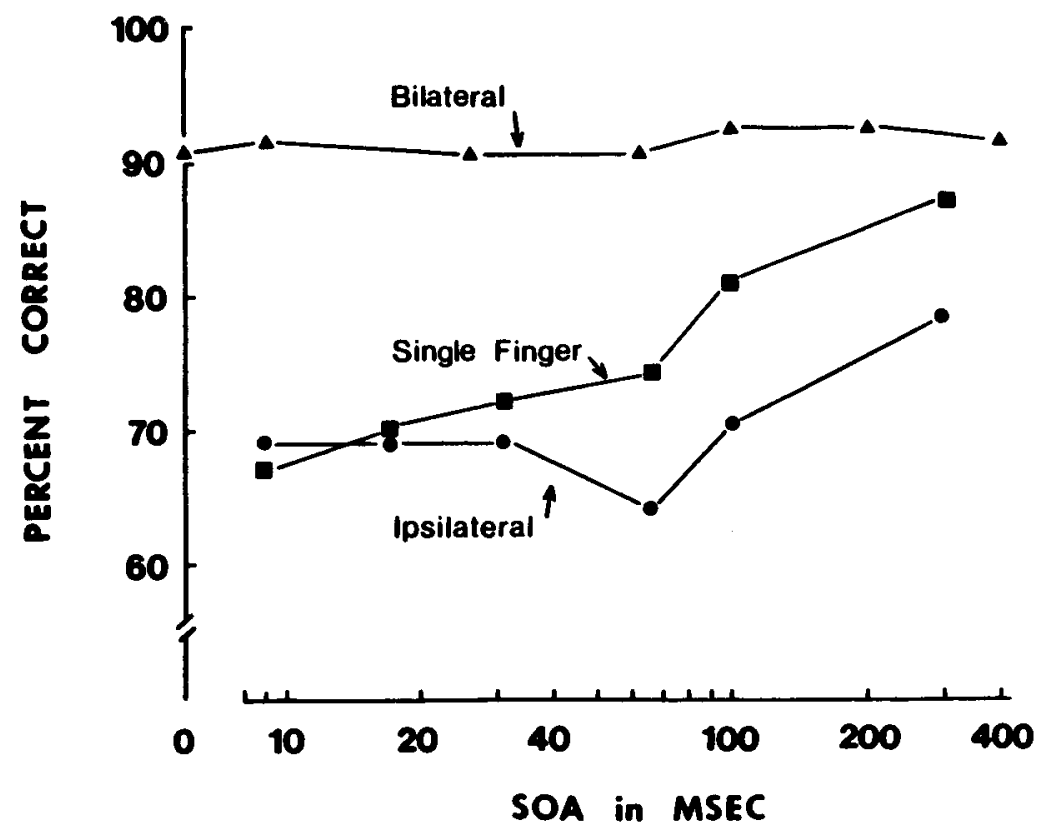

Figure 15. Percent correct in a discrimination task as a function of SOA. The singlefinger and ipsilateral data are from Craig (1983). Ten patterns were tested.

to one of 400 msec shows that performance is improving. Additional measures will be necessary to determine if this is a reliable effect or not.

For the bilateral measurements, the arrays contacted the left and right index fingers, whereas for the ipsilateral measurements, the arrays contacted the middle finger and index finger on the left hand. The question might be raised as to whether the bilateral advantage might simply be the result of the change in location of one of the arrays from a middle finger, specifically the left middle finger, to a more sensitive finger, the right index finger. The bilateral directed-/divided-attention results from Experiment 4, in which the two arrays were placed against the left middle and right index fingers, suggest that the bilateral advantage is not the result of the particular fingers used. To be certain that in the discrimination measurements the change in location from a middle finger in the ipsilateral condition to an index finger in the bilateral condition was not the reason for the improved discriminability in the bilateral condition, an additional set of discrimination measurements was made. Using the 10 easy patterns, the arrays in the bilateral condition were placed in contact with the subjects' left middle and right index fingers. Again, patterns presented bilaterally were found to be easier to discriminate than patterns presented ipsilaterally.

\section{DISCRIMINATION AND RESPONSE BIAS}

For the same-different discrimination results in this paper, performance was reported as a maximum percent correct derived from $d^{\prime}$. Using $d^{\prime}$ as a measure of sensitivity permits one to compare performance levels when different response biases are present, as there were in the present measurements. However, an analysis of such biases may shed some light on the differences between ipsilateral and bilateral pattern perception processes.

It has been reported that there is a substantial bias toward responding "same" in a tactile discrimination task, a bias that diminishes as the time between pairs of patterns increases (Craig, 1983). This bias was observed whether the pairs of patterns were presented to a single finger or to two fingers on the same hand.

To see the extent to which this response bias was evident in the current results, the data from several of the discrimination measures, both ipsilateral and bilateral, are presented in terms of the percentage of trials on which subjects made "same" responses. The 10 easy, ipsilateral results are computed from data collected by Craig (1983) for 52-msec patterns. As Figure 17 shows, subjects in the ipsilateral condition are much more likely to respond "same" than to respond "different" at briefer SOAs. In judging the strength of this response bias, one needs to keep in mind that subjects are informed that half the trials are "same" and half "different" and that subjects are receiving trial-by-trial feedback. ${ }^{2}$ No such response bias appears to exist for patterns presented bilaterally. This is true even in the bilateral letter-discrimination task where performance is relatively poor.

An analysis of the subjects' responses shows that over the range of SOAs from 0 to $100 \mathrm{msec}, \mathrm{d}^{\prime}$ remains unchanged for the ipsilateral conditions (and, indeed, for all bilateral conditions, including SOAs beyond $100 \mathrm{msec}$ ). However, for the ipsilateral condition for SOAs from 0 to $100 \mathrm{msec}$, the hit rate is increasing 


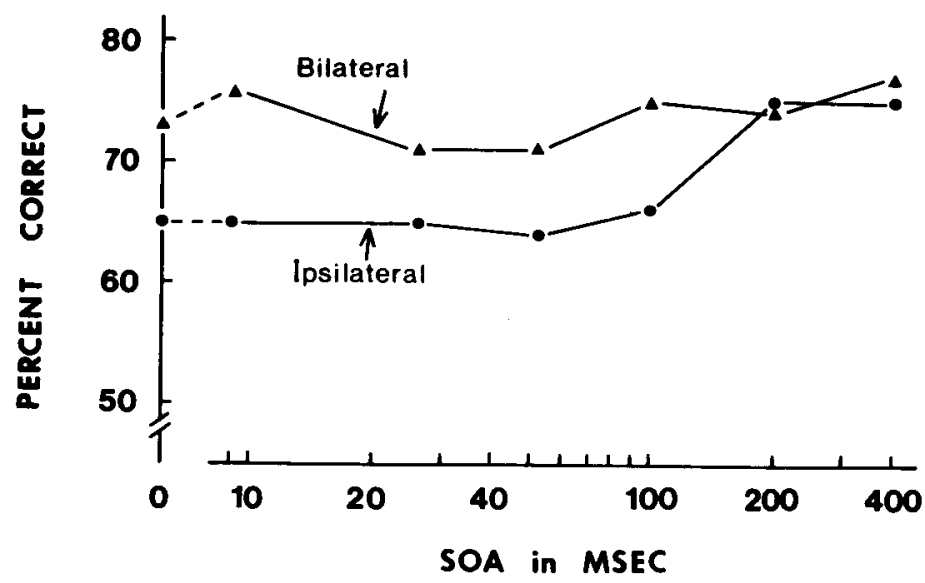

Figure 16. Percent correct in a discrimination task as a function of SOA. Twenty-six patterns were tested. The ipsilateral data from Figure 3 are replotted for purposes of comparison.

(differents called different) and the false-alarm rate (sames called different) is also increasing. It is as though, with no change in sensitivity, all pattern pairs feel more different to the subjects with increasing SOA. For bilaterally presented patterns, sensitivity remains unchanged with SOA, as do the hit and false-alarm rates.

The ipsilateral responses presented in Figure 17 suggest that there may be some sort of "built-in" bias toward responding same at brief SOAs. Might this bias result from the inputs usually received in the course of haptic exploration? As the hand is moved across an object, the probability of two fingers' encountering the same feature or pattern is much greater at briefer SOAs than it is at longer SOAs. Consider a person running his or her index and middle fingers along the serrated edge of a key. The index finger encounters the first "hill" on the key and this is followed shortly by the middle finger's moving across the same hill. As the fingers move on to scan additional hills and valleys, the time elapsing from the presentation of the first hill to the middle finger increases and the probability of the index finger's encountering an identical hill drops and, except in the instance of some sort of recurrent pattern, remains at a constant low level.

In bilateral haptic exploration, the two hands are rarely held adjacent to one another; rather, the two hands are usually exploring different aspects of an object. It is much less likely for two fingerpads on opposite hands to encounter the same patterns at very brief SOAs, leading to the speculation that there would be no built-in bias to respond "same" for bilateral patterns. Such speculations leave unanswered the asymmetry between "same" and "different" responses; that is, there is less of a bias toward responding "different" at longer SOAs for ipsilateral patterns and no bias at any SOA for bilateral patterns.

\section{GENERAL DISCUSSION}

Can the results of these experiments be attributed to attentional mechanisms, or can they be accounted for by some other process, such as masking? Masking is an attractive alternative because it is a pervasive phenomenon on the skin with patterns of the type that were used in these experiments and because some of the characteristics of masking are similar to the characteristics that are attributed to attention (Craig, 1978, 1980, 1983). Looking first at the ipsilateral results, the time course of forward and backward masking is similar to that of the functions shown in Figures 3 and 4. If masking were responsible for these functions, it would be expected that performance in a discrimination task would be poor at brief SOAs and improve as SOA increased. Similarly, if the difficulty in the divided-attention conditions was that the two patterns masked one another, then performance might well improve with increasing SOA.

There are difficulties, however, in attributing the ipsilateral results to masking. First, if masking were responsible for the directed-/divided-attention results, then performance levels should be lowest with the briefest SOAs. This is the case with the divided-attention condition, but in the directed-attention condition, with an SOA of 0 msec, performance levels are well above those of the divided-attention condition. It is generally held that masking cannot be reduced or eliminated by simply instructing the subject to ignore the irrelevant (masking) stimulus. In fact, the instructions to subjects in masking studies are precisely to attend to the target and ignore the masker. Thus, masking is unlikely to explain the differences between the directed and divided conditions, a difference we would like to attribute to attention. Second, with regard to the combined pattern measurements, the best perfor- 


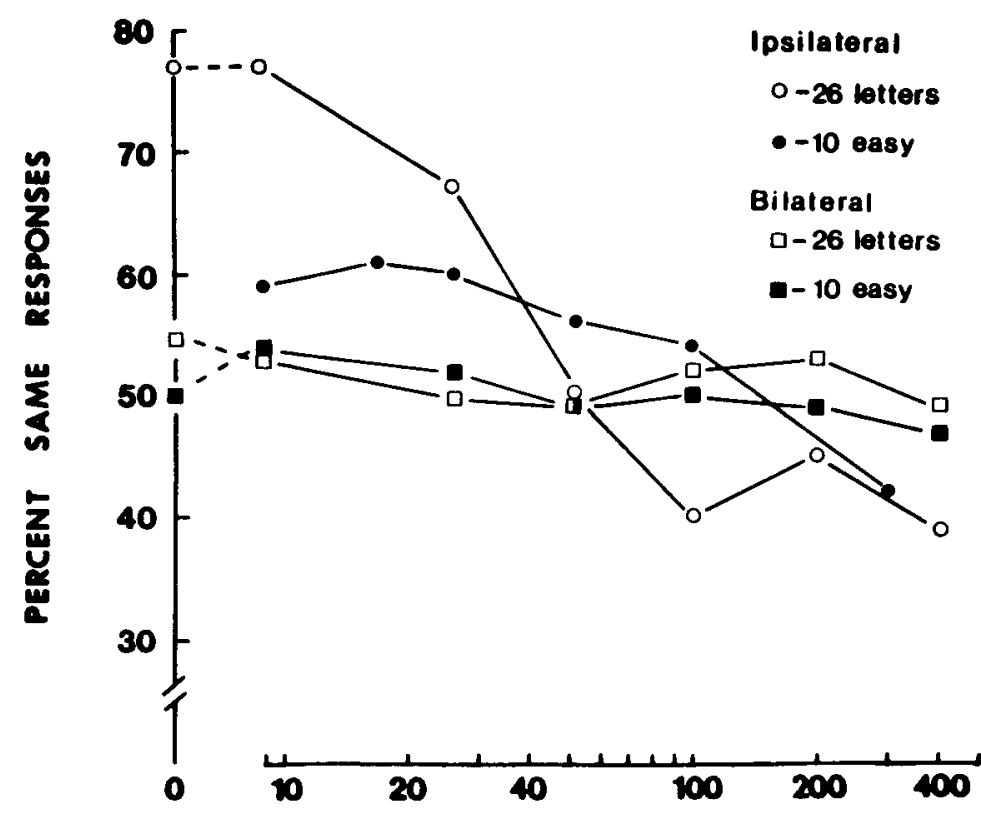

SOA in MSEC

Figure 17. Percent "same" responses in a discrimination task as a function of SOA. Pattern pairs were presented either ipsilaterally or bilaterally, and two different pattern sets were used, 10 easy patterns and 26 letters.

mance was obtained when the two halves of the patterns were presented to the same finger, a condition that will produce more masking than will the presentation of the two halves to separate fingers (Craig, 1983).

Can the difference between the ipsilateral and bilateral results be attributed to masking? On the basis of detection masking results (Gescheider, Herman, \& Phillips, 1970; Gilson, 1974; Sherrick, 1964; Snyder, 1977), it would be expected that ipsilateral presentation of two patterns would produce greater interference than bilateral presentation. The results suggest that part of the difference between ipsilateral and bilateral results in the directed-/divided-attention measurements may be due to masking. Figure 11 shows that performance is better in the bilateral-directed condition than in the corresponding ipsilateral condition, an ipsilateral decrement of about $6.5 \%$. Presumably, this decrement is the result of more mutual interference, masking, when the two patterns are presented to the same hand; however, the amount of masking is not great enough to account for the differences between the two functions. Thus, reduced masking may account for part, but not all, of the improvement seen when patterns are presented bilaterally in the directed-/dividedattention measurements.

For the bilateral discrimination measurements, the ordering of results across conditions is not entirely consistent with an explanation based on masking. Reduced masking is likely to accompany a shift from ipsilateral to bilateral pattern presentation and would lead one to predict better bilateral than ipsilateral discrimination performance, as shown in Figures 15 and 16. However, it has also been shown that there is considerably more temporal masking for patterns presented to a single finger than for ones presented to two fingers on the same hand. Indeed, it was this observation that led us to suggest an attentional explanation for the relatively poor performance in the ipsilateral condition (Craig, 1983). A masking explanation would reverse the ordering of performance levels for the ipsilateral and single-finger functions shown in Figure 15.

In a masking paradigm, subjects are instructed to ignore one stimulus and attend to another, whereas in the present study of attention, subjects are instructed to attend to both stimuli. Although masking, at least when conceived of as a reduction in target recognition in the presence of another pattern, is unlikely to be the sole process to account for the present results, it is clear that there are parallels between masking and particularly the directed-/divided-attention paradigm. In both paradigms, two stimuli are presented and a decrement in performance is measured. One might view masking between two sites of stimulation as representing the inability to focus attention entirely on the target site, and the attentional deficit as representing the inability to divide attention between two sites. The two processes, masking and attention, may represent two aspects of the skin's ability to organize patterns spatially. Also, viewing masking and attention in this way suggests that bilateral presentation of two pat- 
terns permits much greater flexibility than does ipsilateral presentation: Subjects may either attend to a single site, no masking, or attend to two sites with little attentional deficit.

What do these experiments tell us about how information is combined from separate fingers? First, it needs to be emphasized that these reults show that the skin can process spatial patterns presented to two fingers simultaneously. The present results are consistent with a view of tactile attention that subjects can attend to more than one site of stimulation at a time but that dividing attention results in some loss in processing spatial patterns at a particular site. Furthermore, subjects can focus their attention on a particular site with a concomitant improvement in the processing of a pattern presented to that site.

Earlier work has shown that it is the time between pattern onsets that is the critical temporal dimension in forward and backward masking and in discrimination tasks (Craig, 1983). When patterns are presented successively to the same site on the skin, performance in both masking and discrimination paradigms improves as SOA increases (Craig, 1983, 1985). These earlier observations, coupled with the present results, suggest that when a single hand is used to explore an object, in addition to bringing other facets of the object into contact with the fingers, the sequence of finger movements across the object may serve some other functions. Specifically, these movements may serve to separate temporally the onsets of the patterns arriving at each finger and thereby to reduce masking between fingers. The present results also suggest that this temporal separation, provided it is greater than $100 \mathrm{msec}$, should also enable an individual to attend to the fingers separately. According to this speculation, the exploration of an object by the hand would serve not only to bring the fingers into contact with the varied surfaces of the object and to engage kinesthetic mechanisms, but also to improve the clarity of the patterns received at each finger. The latter improvement would be the result of (1) reduced.masking, particularly on the same finger (Craig, 1978, 1983), and (2) increased ability to switch attention from one finger to another.

The present experiments were undertaken, in part, to see if the number of patterns that could be processed in a brief period of time could be increased. In an attempt to avoid some of the problems of temporal masking, the patterns were presented to two fingers, but that procedure introduces another problem-attention (Craig, 1985). It now appears that for certain tasks attentional deficits can be reduced, and in some cases eliminated, if the patterns are presented bilaterally. The discrimination task shows this most clearly, with bilateral performance being better than either ipsilateral (two fingers) or single-finger performance (Figure 15). The same is clearly true for the ipsilateral/bilateral conditions in the divided-attention tasks. If both members of the pair of patterns were presented to the same finger in close temporal proximity and subjects were required to identify one of the patterns, the result would reflect a paradigm similar to that of tem- poral masking. Because temporal masking is so much greater when the patterns are presented to the same finger (Craig, 1983), one would predict that if the directed-/ divided-attention measures at briefer SOAs were repeated and a single finger condition included, low levels of performance would be produced due to masking. Furthermore, one would predict little difference between directed- divided-attention conditions, because the subject need not shift attention from the same spatial location.

We tested these predictions in a directed-/dividedattention task with pairs of patterns presented to a single finger, to two fingers on the same hand, or to two fingers on opposite hands. The patterns were presented for $52 \mathrm{msec}$ with a 52-msec SOA. With the single-finger condition, there were no differences in performance resulting from whether the instructions-regarding which of the two patterns (the first or the second) they were to reportwere given to the subjects before (directed attention) or after the pattern had been presented (divided attention). Not surprisingly, the best performance was achieved in the bilateral directed condition; this was followed by the ipsilateral directed condition, and then by the bilateral divided condition. Performance levels in the ipsilateral divided-attention condition were approximately equal to those in both single-finger conditions. The latter result is somewhat surprising, since it suggests that attentional deficits may in some cases rival deficits due to masking, and the measurements, therefore, need to be replicated with a greater number of patterns.

The present experiments suggest that one reason for Lappin and Foulke's (1973) observation that braille cell perception was better with two fingers on opposite hands is that it is easier to attend to two fingers bilaterally situated. In this regard, it is noteworthy that Tadoma users typically use a single hand (Reed et al., 1982). However, it has been observed that if they are having difficulty in understanding someone, they may place a second hand on the speaker's face, a response that seems to improve their performance (N. I. Durlach, personal communication, 1983). As noted before, we have also observed in our laboratory that with tactile patterns derived from speech signals (Craig et al., 1985) and presented to the fingerpads by means of two tactile arrays, performance is consistently better if bilateral rather than ipsilateral fingers are used.

The present experiments and the above observations suggest that designers of cutaneous communication systems should consider making deliberate use of bilateral stimulation. The present studies have been limited to the fingerpads. Further work, both applied and basic, needs to be directed to the nature of ipsilateral and bilateral attentional mechanisms when sites other than the fingers are involved.

\section{REFERENCES}

Buss, J. C., Katcher, M. H., Rogers, C. H., \& Shepard, R. P. (1970). Optical-to-tactile image conversion for the blind. IEEE Transactions on Man-Machine Systems, MMS-11, 58-64. 
Cholewiak, R. W., \& CRaIG, J. C. (1984). Vibrotactile pattern recognition and dicrimination at several body sites. Perception \& Psychophysics, 35, 503-514.

Craig, J. C. (1968). Vibrotactile spatial summation. Perception \& Psychophysics, 4, 351-354.

CraIG, J. C. (1978). Vibrotactile pattern recognition and masking. In G. Gordon (Ed.), Active touch - the mechanism of recognition of objects by manipulation: A multi-disciplinary approach. Oxford: Pergamon Press.

CraIG, J. C. (1980). Modes of vibrotactile pattern generation. Journal of Experimental Psychology: Human Perception \& Performance, 6 , 151-166.

CraIG, J. C. (1981). Tactile letter recognition: Pattern duration and modes of pattern generation. Perception \& Psychophysics, 30, 540-546.

CraIG, J. C. (1983). The role of onset in perception of sequentially presented vibrotactile patterns. Perception \& Psychophysics, 34, $421-432$

Craig, J. C. (1985). Tactile pattern perception and its perturbations. Journal of the Acoustical Society of America, 77, 238-246.

Craig, J. C., Green, B. G., \& Rhodes, R. P. (1985). Ipsilateral vs. bilateral placement of a tactile vocoder display. Joumal of the Acoustical Society of America, 77, 1266-1268.

Franzen, O., Markowitz, J., \& SwETs, J. A. (1970). Spatially-limited attention to vibrotactile stimulation. Perception \& Psychophysics, 7, 193-196.

GelDard, F. A., \& Sherrick, C. E. (1965). Multiple cutaneous stimulation: The discrimination of vibratory patterns. Joumal of the Acoustical Society of America, 37, 797-801.

Gescheider, G. A., Herman, D. D., \& Phillips, J. N. (1970). Criterion shifts in the measurement of tactile masking. Perception \& Psychophysics, 8, 433-436.

GiLson, R. D. (1968). Some factors affecting the spatial discrimination of vibrotactile patterns. Perception \& Psychophysics, 3, 131-136.

Gilson, R. D. (1974). Vibratory masking. In F. A. Geldard (Ed.), Cutaneous communication systems and devices. Austin, TX: Psychonomic Society.

Green, B. G., Craig, J. C., \& Pisoni, D. B. (1983). Vibrotactile communication of information about consonants: Vowels mask consonants. Perception \& Psychophysics, 33, 507-515.

Green, B. G., Craig, J. C., Wilson, A. M., Pisoni, D. B., \& Rhodes, R. P. (1983). Vibrotactile identification of vowel spectra. Journal of the Acoustical Society of America, 73, 1766-1778.
HILL, J. W. (1974). Limited field of view in reading lettershapes with the fingers. In F. A. Geldard (Ed.), Cutaneous communication systems and devices. Austin, TX: Psychonomic Society.

Kahneman, D. K. (1973). Attention and effort. Englewood Cliffs, NJ: Prentice-Hall.

LAPPIN, J. S., \& FoulKE, E. (1973). Expanding the tactual field of view. Perception \& Psychophysics, 14, 237-241.

McFADDEN, D. (1970). Three computational versions of proportion correct for use in forced-choice experiments. Perception \& Psychophysics, 8, 336-342.

Reed, C. M., Durlach, N. I., \& Braida, L. D. (1982). Research on tactile communication of speech: $A$ review (ASHA Monograph No. 20). Rockville, MD: American Speech-Language-Hearing Association.

SHERRICK, C. E. (1964). Effects of double simultaneous stimulation of the skin. American Journal of Psychology, 77, 42-53.

Shiffrin, R. M., Craig, J. C., \& Cohen, E. (1973). On the degree of attention and capacity limitation in tactile processing. Perception \& Psychophysics, 13, 328-336.

SNYDER, R. (1977). Vibrotactile masking: A comparison of psychophysical procedures. Perception \& Psychophysics, 22, 471-475.

\section{NOTES}

1. The percent correct, assuming that subjects could not attend to more than one finger, was calculated by multiplying the percent correct in the directed condition, 93 , by .5 , the proportion of trials on which subjects would be attending to the "correct" finger plus $10 \%$, chance performance on the unattended finger ( 10 possible patterns), multiplied by .5 , the proportion of trials on which the subject would be attending to the "incorrect" or nonrequested finger, $(93)(.5)+(10)(.5)=52 \%$.

2 . To demonstrate the role feedback plays in discrimination, we tested three subjects with patterns presented to the same finger. The subjects were told that half the pairs were the same and half different, but they received no trial-by-trial feedback. At an SOA of $9 \mathrm{msec}, 90 \%$ of the subjects' responses were "same." This percentage dropped to $48 \%$ by the time the two patterns were separated by $56 \mathrm{msec}$.

(Manuscript received February 27, 1985; revision accepted for publication September 30, 1985 .) 